\title{
PROCESOS EDUCATIVOS MEDIADOS POR PLATAFORMAS VIRTUALES EN LA METODOLOGÍA PRESENCIAL USANDO bLEARNING
}

\section{EDUCATIONAL PROCESSES MEDIATED BY VIRTUAL PLATFORMS IN CLASSROOM ME- THODOLOGY USING bLEARNING}

Edgar Javier Carmona Suarez ${ }^{1}$ y Lina María Gallego Berrio ${ }^{1}$

Programa de Licenciatura en Matemáticas, Universidad del Quindío. Colombia. ecarmona@uniquindio.edu.co,linag@uniquindio.edu.co.

Recibido: Enero 17 de 2012

Aceptado: Febrero 2 de 2012

*Correspondencia del autor. Facultad de educación, Programa de Licenciatura en Matemáticas, Universidad del Quindío, Armenia (Quindío); correo electrónico: ecarmona@uniquindio.edu.co

\section{RESUMEN}

Este proyecto pretende determinar el impacto de los procesos educativos mediados por plataformas educativas virtuales en algunos cursos de la metodología presencial, usando bLearning o aprendizaje mixto. Para este fin, se toma como base, analizar el estado de la educación virtual de la Universidad del Quindío.

Inicialmente se hizo una caracterización de los estudiantes de la universidad, lo que permitió conocer su disposición hacia el eLearning, mediante el uso de la Plataforma educativa Moodle. Luego se realizó un estudio sobre la experticia de los profesores en el uso de las herramientas informáticas y las mediaciones tecnológicas como apoyo a la docencia; para ello se tomó una muestra aleatoria de 144 profesores de la cual se extrajo un grupo de experimentación con 18 profesores que orientaban 22 cursos de la universidad a los cuales asistieron 600 estudiantes. Esta información junto con los datos recolectados de las preferencias y la visión de los estudiantes proporcionó un panorama de la forma como se han desarrollado los procesos de virtualización en la Universidad.

Finalmente, como producto de esta investigación se implementaron 22 cursos en Moodle, lo que permitió conocer las necesidades de formación y desarrollo de competencias en ambientes virtuales de aprendizaje de los profesores y estudiantes. De igual manera se mostró que es posible fortalecer los procesos de enseñanzaaprendizaje y de comunicación mediante la utilización del bLearning.

Palabras clave: Educación virtual, bLearning, eLearning, Moodle. 


\begin{abstract}
:
This project aimed to determine the impact of educational processes mediated by virtual educational platforms in some classroom methodology courses, using bLearning or blended learning process. Based on this, it was proposed to show the status of virtual education at the University of Quindío.

A deep study carried out with the students measured their sensitivity towards eLearning, using the Moodle learning platform and a study on the domain of computer tools by teachers, using a random sample of 144 teachers determined the use of technological tools as a support to academic work in 2010. Also, there was created a working and experimentation group, with 18 teachers responsible for 22 courses attended by 600 students. This collected information along with the preferences and vision of the students provided an overview of how the virtual processes have been developed at the University.

The product of this investigation, 22 courses in the Moodle platform, allowed us to know the needs of training and skills in virtual learning environments for teachers and students who participated. Besides, it was demonstrated that it is possible to strengthen the teaching and learning processes and communication through the implementation of B-Learning.
\end{abstract}

Key words: virtual education, bLearning, eLearning, Moodle.

\section{INTRODUCCIÓN}

En la actual sociedad se observan transformaciones en todas las actividades del ser humano tanto individuales como organizacionales, que conducen a una nueva naturaleza del trabajo (Rubio Royo, 2004) y una virtualización creciente en todos los procesos (Druker F, 1999). Ante este panorama de cambios permanentes, los individuos se enfrentan a nuevos paradigmas, algunos de estos se relacionan con el aprendizaje. Es así como prosperan las organizaciones emergentes, según la UNESCO (2005) surgen formas alternas de aprender (eLearning), de enseñar (eTeaching), de conocer (e-conocimiento), las relaciones interpersonales se transforman (networking y redes sociales), el trabajo evoluciona (teletrabajo, knowledge-working), la formación permanente toma mayor relevancia (LLL-Long live learning), y en general se observa una dinámica social cada vez más ligada al mundo digital.

La universidad debe entonces adaptase a estos nuevos escenarios. Frente a un sistema en el que ha roto las barreras espacio-temporales, debe ofrecer otras alternativas para cumplir con las demandas de formación y capacitación de la sociedad. La Educación Virtual se ha convertido entonces en una herramienta que resuelve algunos de los problemas que enfrentan las instituciones educativas. Estos procesos virtuales se están enfocando en la Universidad del Quindío a través de las tres metodologías de formación: presencial, a distancia y virtual. Las Tecnologías de la Información y la Comunicación
(TIC) por naturaleza son el principal instrumento o herramienta para educación en línea, mientras que tanto en la metodología a distancia como en la presencial es posible a través del uso de las TIC implementar procesos blended learning (bLearning o mixtos).

Esta investigación pretende fomentar la implementación de procesos virtuales en la educación presencial en la Universidad del Quindío, con ello ofrecer alternativas para el profesor a la hora de estructurar sus procesos pedagógicos y al estudiante formas alternas de aprendizaje mediadas por TIC y reducir el problema de disponibilidad de espacios físicos en la Institución.

\section{CONTEXTO}

Se tomó como punto de partida trabajos y desarrollos realizados en la Universidad del Quindío ${ }^{1}$, enfatizando en la intencionalidad del establecimiento de procesos bLearning como estrategia para el mejoramiento de los procesos académicos. El análisis y foco central se orienta articular los elementos conceptuales y una propuesta de desarrollo, de tal manera, que se pueda mejorar el desempeño del profesor en ambientes caracterizados por el trabajo en red y la gestión del conocimiento. Con este foco, los conceptos que han sido objeto de estudio son:

\footnotetext{
${ }_{1}^{1}$ Proyecto del Aula Virtual, Proyecto de la Unidad de Virtualización, Lineamientos Pedagógicos para la formación virtual, Plan de Desarrollo Institucional, entre otros.
} 
- La Educación Virtual entendida como un conjunto de posibilidades, expectativas y ventajas que se suman a los sistemas tradicionales educativos, según aportes de autores como Bulchand 2002, Murphy 2003 y Fidalgo 2003.

- Nativos digitales, emigrantes digitales y multitudes inteligentes (Rheingold, 2004, Piscitelli 2006)

- La formación en la modalidad eLearning y bLearning.

- Diseño de cursos en plataformas virtuales (Learning Management System, LMS), sistemas que hicieron de la informática e internet un aliado para generar conocimientos y aprendizajes y que se van convirtiendo en herramientas para los docentes.

- Los procesos educativos y programas apoyados por las TIC, así como las tendencias en formación virtual en la Universidad del Quindío.

Los educadores tienen múltiples razones para aprovechar el amplio espectro de posibilidades que proporcionan las TIC e impulsar un paradigma educativo más personalizado y centrado en el estudiante. Según Marqués (2008), además de la necesaria alfabetización digital de los estudiantes y el aprovechamiento de las TIC para mejorar la productividad en general, existen consideraciones como el alto índice de fracaso escolar y la creciente multiculturalidad de la sociedad que constituyen poderosas razones para aprovechar las posibilidades de innovación metodológica que ofrecen las TIC, logrando una escuela más eficaz e inclusiva (Marqués Graells, 2008).

Las principales funcionalidades de las TIC en la educación, en mayor o menor medida, están relacionadas con: alfabetización digital, gestión del conocimiento, procesamiento de datos, administración de centros educativos, mediador de procesos de enseñanza y aprendizaje (motivar, informar, entrenar, guiar aprendizaje, evaluar), comunicación (consultas, gestiones, notificaciones, orientaciones), mejorar la interrelación entre profesores a través de redes y comunidades virtuales, compartir recursos y experiencias, facilitar el trabajo colaborativo, contribuir a la formación continua (en todo momento y lugar) y como medio lúdico para el desarrollo cognitivo.

El problema de cómo integrar la tecnología en la educación, conduce al uso de los ambientes Web para el aprendizaje. Es necesario plantearse: ¿Cómo llegar a los estudiantes?, ¿Son pertinentes los contenidos?, ¿Cómo lograr espacios que simulen la vida real y potencien el aprendizaje? Estos interrogantes no tienen una respuesta trivial, por lo que el docente debe estar en procura de mejorar el quehacer en función de generar aprendizaje propios y de los estudiantes.

El sistema de enseñanza digital o eLearning presenta problemas como: ausencia de contacto humano que dificulta el sentirse parte de la comunidad, altos niveles de deserción, falta de motivación y autodisciplina, requiere de habilidades de lecto-escritura digitales, patrones culturales que asocian aprendizaje con profesor y educación con centros educativos.

Lo anterior lleva a buscar nuevas alternativas para el aprendizaje, es así como surge el blended Learning, que combina la enseñanza presencial con la virtual, "which combines face-to-face and virtual teaching” (Coaten, 2003; Marsh, McFadden, \& Price, 2003). Una idea clave es la selección de los medios adecuados para cada necesidad educativa. Brennan señala que el término tiene diferentes significados para diferentes personas: "cualquier posible combinación de un amplio abanico de medios para el aprendizaje...” (Brennan, 2004).

\section{ANTECEDENTES}

Estos temas ya se han venido estudiando en la Universidad del Quindío en razón a que la educación virtual ha sido un renglón importante en los planes de desarrollo. Dos dependencias han trabajado directamente en esta área: el Aula Virtual de la Facultad de Educación y la Unidad de Virtualización como dependencia adscrita a la Vicerrectoría Académica.

En el año 2000 como una iniciativa de la Facultad de Educación en la que se recogía la experiencia del programa de Ciencias de la Información y la Documentación Bibliotecología y Archivística y las políticas del $\mathrm{MEN}^{2}$, se creó el Aula Virtual, grupo integrado por cinco docentes de la misma Facultad (Carmona Suárez \& Gallego Berrío, 2008). Durante los diez años siguientes, el proyecto Aula Virtual se encargó de la formación de docentes mediante dos diplomados en TIC. Uno de los requisitos para que los estudiantes (profesores uniquindianos) finalizaran el diplomado era la realización de un cursoWeb. Fue así, como se empezó a generar un repositorio de recursos multimediales y cursos virtuales.

Una vez terminados los cursos, fue necesario llevarlos a

${ }^{2} \overline{\text { Particularmente con el ofrecimiento del programa de formación MAES- }}$ TRO 1.0 
un servidor para ser centralizados; se requirió entonces el uso de un LMS (Sistema Gestor de Aprendizaje), término para entonces novedoso. En este sentido, la Universidad adelantó conversaciones con los distribuidores de WebCt y Blackboard, negociación que fracasó por los costos de la utilización de este software.

En el año 2003 y como una experiencia de uso de software libre, se instaló en el Grupo de Investigación y Desarrollo de Software (GEDES) una versión de la Plataforma Claroline, con ello se inició de manera oficial el uso de plataformas para impartir cursos en la Institución. Sin embargo, en el 2004 se instaló una versión de prueba de Moodle y en el 2006 la versión definitiva, programa apenas conocido, pero con mucha proyección. Se han hecho varias actualizaciones, siendo la 1.9.12 la última la versión.

La Universidad empezó a organizar su estructura académica administrativa en materia de virtualidad y hacer extensiva a toda la Institución la labor que venía realizando el Aula Virtual. Para ello, en el 2008, se crea la Unidad de Virtualización. Su misión fue mantener en continua modernización, actualización y desarrollo del modelo de educación con apoyo de las TIC (eLearning), como una alternativa para ampliar la oferta educativa y cobertura; apoyar la docencia y el aprendizaje presencial, a distancia y virtual, con herramientas que optimicen la interacción y las interactividades en los procesos de enseñanza aprendizaje.

La Unidad de Virtualización, normaliza y regula todas las acciones en virtualidad en la Institución. Así lo muestran documentos sobre la política en este sentido, tales como "la Educación virtual en la Universidad del Quindio. Dimensión pedagógica” (Díaz, 2008) e "Incorporación de las TIC en la Universidad del Quindio. Políticas y estrategias" (Londoño Orozco \& Díaz Jurado, 2008). Se destaca que la educación virtual se concibe como un sistema que gira alrededor de cuatro ejes fundamentales: tecnológico, pedagógico, comunicativo y organizacional (Díaz, 2008), tomando como marco referencial el Plan de Desarrollo Institucional y el Plan Decenal de la Educación 2006-2016, específicamente en el uso de las TIC en la Educación.

En el año 2010, el Consejo Académico de la Universidad, para institucionalizar el uso del eLearning en la Universidad del Quindío, definió los mecanismos y criterios para el diseño, acceso e implementación de la metodología virtual y los recursos de apoyo a la pre- sencialidad y distancia. De esta manera se consolida la metodología virtual en la Institución y se define Moodle como plataforma para ofrecer cursos virtuales y presenciales apoyados por las TIC. Para diciembre de 2006, el servidor de Moodle de la Universidad contaba con cerca de 5000 usuarios registrados, 654 cursos de los cuales 150 están operativos y el resto en periodo de construcción. Cifras que en diciembre del 2011 ya se habían duplicado.

Como parte de la política de la formación profesoral, se ha formalizado la capacitación en diseño de Ambientes Virtuales de Aprendizaje (AVA). En este programa se han capacitado cerca de 400 profesores, los cuales han construido sus cursos virtuales y se han puesto a disposición de la comunidad universitaria los cursos aprobados por los consejos curriculares.

Actualmente, la Universidad oferta tres programas en la metodología virtual: Ciencias de la Información Bibliotecología y Archivística, Técnico Profesional en Servicios de Recepción y Alojamiento y Bebidas, Tecnología en Gestión de Empresas Turísticas, al igual que diferentes acciones de formación dirigidas a profesores y un banco de objetos virtuales de aprendizaje (OVA).

\section{MÉTODO UTILIZADO}

Se optó por un método flexible, de base cualitativa, que incorpora entrevistas a miembros de Comunidad Universitaria (autoridades académicas, profesores, estudiantes y administrativos) y dinámicas de trabajo de colaboración con los profesores; además, análisis de documentos institucionales, páginas Web y planes específicos de uso de las TIC. Ello demandó la ejecución de acciones como:

- Documentación y análisis de un marco conceptual.

- Elaboración de un plan de acción sobre cómo abordar el uso de sistemas bLearning en la Universidad del Quindío.

- Sensibilización, motivación y capacitación a 40 profesores de diferentes programas. De este grupo, se conformó un equipo de trabajo con 18 docentes voluntarios, con ellos se hizo un diagnóstico inicial para determinar el dominio de los docentes en el manejo de las TIC. Con esta formación y con las asesorías individuales, se construyeron e implementaron cursos en la Plataforma Moodle. En la etapa siguiente los profesores desarrollaron sus cursos utilizando la estrategia blearning para el trabajo con los estudiantes. 
- La población objeto de estudio estuvo conformada por estudiantes y profesores. Se seleccionaron muestras a las que se aplicaron los instrumentos diseñados así: para el diagnóstico inicial a 190 estudiantes y 144 docentes de los diferentes programas y se entrevistaron siete (7) directivas académicoadministrativas. Se conformó un grupo de trabajo y experimentación, en el que participaron 18 docentes que orientaban 22 cursos a los cuales asistieron 600 estudiantes. Finalmente, seleccionó una muestra de 298 estudiantes para determinar el impacto producido por la implementación de procesos bLearning en la Universidad.

\section{RESULTADOS Y DISCUSIÓN}

Partiendo del objetivo planteado, el grupo investigador profundizó en temas que permitieran obtener un panorama sobre la problemática relacionada con la implantación de procesos de educación virtual. En ese orden de ideas, surgieron interrogantes: ¿Cómo ofrecer a los profesores un sistema de formación utilizando la Web que sirva de apoyo a la práctica del docente, para mejorar su productividad en actividades personales, de docencia, investigación y extensión universitaria?, ¿Cómo implementar una estrategia de formación utilizando procesos bLearning de tal manera que se logre mejoras en los procesos educativos en la Universidad del Quindío? (Carmona Suárez \& Gallego Berrío, 2008).

Plantear alternativas de solución a estos problemas, conlleva a la discusión de la forma como los profesores se enfrentan a las olas de cambio permanente que caracterizan a la sociedad. Es claro y evidente la necesidad de usar las TIC y que sin ellas algunos problemas serían difíciles de resolver. Pero tan importante como su uso, es saber cuáles, en qué medida, en qué momento y cómo apropiarlas. En este sentido nuestra sociedad actual dispone de arquitecturas tecnológicas, conectividad, instrumentos para la gestión del conocimiento, herramientas Web 2.0, plataformas educativas y redes sociales, entre otras herramientas. Oferta que cada vez se amplía mas, dada la importancia y el protagonismo que el software libre nos provee. Se ha llegado a la situación tal, que al momento de emprender un proyecto académico, comunicativo u organizacional, se dispone de varias herramientas libres (Carmona, 2008).

Los estudiantes actuales se catalogan en lo que se ha denominado "nativos digitales"3, sin detallar en las connotaciones de esta expresión, se propuso caracteri- zar a los estudiantes y la forma como ellos utilizan el computador y los recursos informáticos. En este sentido se encontró que el $79 \%$ de la población estudiantil uniquindiana es menor de 25 años, lo que indica que nacieron después de la invención del PC, de la creación de Internet y de las tecnologías móviles. Por su parte, el $41 \%$ de los profesores de la Universidad, tienen una antigüedad laboral mayor de 12 años, lo que deja entrever que tenemos una población muy joven, atendida por una planta docente mayor, lo que traduce la existencia de una diferencia generacional, donde para reducir esta brecha digital se deben establecer acciones de formación.

Cuando se preguntó a los profesores sobre la capacitación recibida en el uso de las TIC y temas relacionados, el $41 \%$ afirmó recibirla de la Universidad. De acuerdo a estos resultados, si se plantea un programa de formación en TIC para los profesores, se tendría un potencial mínimo del 59\%. Sin embargo, el hecho de que los profesores no hayan recibido entrenamiento por parte de esta Institución en el uso de herramientas tecnológicas, no significa que no tengan la competencia, pues algunos la han adquirido empíricamente y otros se han preparado externamente. Pero a la luz de los comentarios expresados por los profesores, se ve la necesidad que tienen de perfeccionarse en estas áreas: "Se requiere el manejo de herramientas informáticas para apoyar los procesos de enseñanza-aprendizaje”, "debemos formarnos en el uso de TIC en los periodos de vacaciones", "esta formación debe ser de carácter obligatoria", "se debe capacitar muy bien a los docentes y estudiantes en el uso de herramientas informáticas antes de usarlas como mediaciones pedagógicas" y, "se debe dar continuidad en las capacitaciones".

Sobre el uso de las herramientas informáticas, según los profesores, el $88 \%$ de ellos manejan Internet en alto nivel, y alrededor del 1\% no usa internet o lo maneja deficientemente. Además los profesores han adquirido la competencia del uso del correo electrónico, la ofimática (Word, Excel, PowerPoint), foros y redes sociales; en cambio expresan dificultades en el manejo del Academusoft ${ }^{4}$, los blogs y la Plataforma Moodle, entre otros. En la tabla 1, se observan los principales inconvenientes que tienen los profesores para involucrar las TIC en sus espacios académicos. Se nota que la disponibilidad de recursos informáticos en los programas de formación también dificulta la implementación de esta

\footnotetext{
Según Alejandro Piscitelli.

${ }^{4}$ Sistema de gestión académico utilizado por la Universidad del Quindío.
} 
metodología.

Tabla 1. Dificultades de los profesores para usar las TIC

\begin{tabular}{lc}
\hline \multicolumn{1}{c}{ Dificultad } & Porcentaje \\
\hline Manejo de la Plataforma Moodle & $32,5 \%$ \\
Disponibilidad de computadores & $22,9 \%$ \\
Falta de tiempo & $15,3 \%$ \\
Manejo de la Web 2,0 & $12,7 \%$ \\
Velocidad del Internet & $10,4 \%$ \\
Falta de elementos en el programa académico & $9,0 \%$ \\
Falta de capacitación & $8,3 \%$ \\
Acceso a programas con licencia & $6,9 \%$ \\
\hline
\end{tabular}

En la tabla 2, se observan las actividades que son susceptibles de llevar a la Plataforma Moodle en los espacios académicos orientados por los profesores encuestados. Se destacan actividades como lecturas y talleres digitales con el $84 \%$ c/u. Una mirada general muestra la potencialidad del uso de la Plataforma en opinión de los profesores, lo que coincide con la entrevista a las directivas académicas en fortalecer el banco de OVAS de la Unidad de Virtualización.

Tabla 2. Actividades que pueden ser llevadas a la Plataforma Moodle

\begin{tabular}{lcc}
\hline \multicolumn{1}{c}{ Actividades } & $\begin{array}{c}\text { No. de } \\
\text { profesores }\end{array}$ & $\begin{array}{c}\text { Porcentaje de } \\
\text { profesores }\end{array}$ \\
\hline a. Lecturas digitales & 122 & $84,7 \%$ \\
b. Talleres digitales & 121 & $84,0 \%$ \\
c. Presentaciones & 112 & $77,8 \%$ \\
d. Tareas & 107 & $74,3 \%$ \\
e. Consultas & 105 & $72,9 \%$ \\
f. Videos & 99 & $68,8 \%$ \\
g. Evaluaciones & 95 & $66,0 \%$ \\
h. Foros & 91 & $63,2 \%$ \\
i. Bibliografia & 91 & $63,2 \%$ \\
j. Encuestas & 73 & $50,7 \%$ \\
k. Fotos & 68 & $47,2 \%$ \\
1. Chat & 61 & $42,4 \%$ \\
m. Glosario & 58 & $40,3 \%$ \\
n. Software & 52 & $36,1 \%$ \\
o. Blog & 48 & $33,3 \%$ \\
p. Wikis & 41 & $28,5 \%$ \\
\hline
\end{tabular}

Asimismo, los 144 profesores encuestados tienen a su cargo 353 espacios académicos, sobre los cuales se preguntó el grado de virtualización al que se podía llegar en cada uno ellos. (Tabla 3).
Tabla 3. Posibilidades de virtualización de espacios académicos

\begin{tabular}{cc}
\hline $\begin{array}{c}\text { Porcentaje de virtualización posible } \\
\text { en los espacios académicos, según } \\
\text { docentes }\end{array}$ & $\begin{array}{c}\text { Número de } \\
\text { espacios } \\
\text { académicos }\end{array}$ \\
\hline $0 \%$ & 20 \\
{$[1 \%-10 \%]$} & 21 \\
$(10 \%-20 \%]$ & 55 \\
$(20 \%-30 \%]$ & 69 \\
$(30 \%-40 \%]$ & 18 \\
$(50 \%-60 \%]$ & 78 \\
$(70 \%-80 \%]$ & 34 \\
$90 \%$ & 5 \\
$100 \%$ & 53 \\
\hline
\end{tabular}

Se destaca que 78 espacios académicos podrían virtualizarse entre un $50 \%$ y $60 \%$, además 53 asignaturas podrán llegar a ser $100 \%$ virtuales; en contraste 20 espacios académicos no tienen, según los profesores que los orientan, posibilidad de ser llevados a la Plataforma. Sin embargo, al contrastar los resultados de las tablas 2 y 3 , todos los profesores consideraron la posibilidad de virtualizar al menos dos actividades en la Plataforma.

Por otra parte, los estudiantes de la Universidad en la metodología presencial, ya han tomado algunos cursos en forma virtual: Constitución Política, Medio Ambiente, Electiva eLearning, Creatividad Empresarial y Ética. Con respecto a la formación que han recibido, ellos consideran que pueden mejorarse aspectos como: aumento de los recursos y salas informáticas, capacitación, ancho de banda, capacidad de almacenamiento y mejorar el acceso a la Plataforma.

Indagados los estudiantes sobre el uso de la Plataforma Moodle como apoyo en las clases presenciales, opinan que sus profesores desarrollan diversas actividades, entre las cuales se destacan las que presenta la gráfica 1.

La mayor dificultad que se presentan al momento de utilizar recursos virtuales, según los estudiantes es la falta de capacitación en el manejo de las TIC (tabla 4). Nótese la diferencia entre los criterios de los estudiantes, al ser aplicado el mismo instrumento al inicio y al final del periodo observado.

Finalmente, los estudiantes sugieren mejorar la capacidad de almacenamiento y el acceso a la Plataforma Moodle, opinión que coincide con lo expresado por los profesores. 


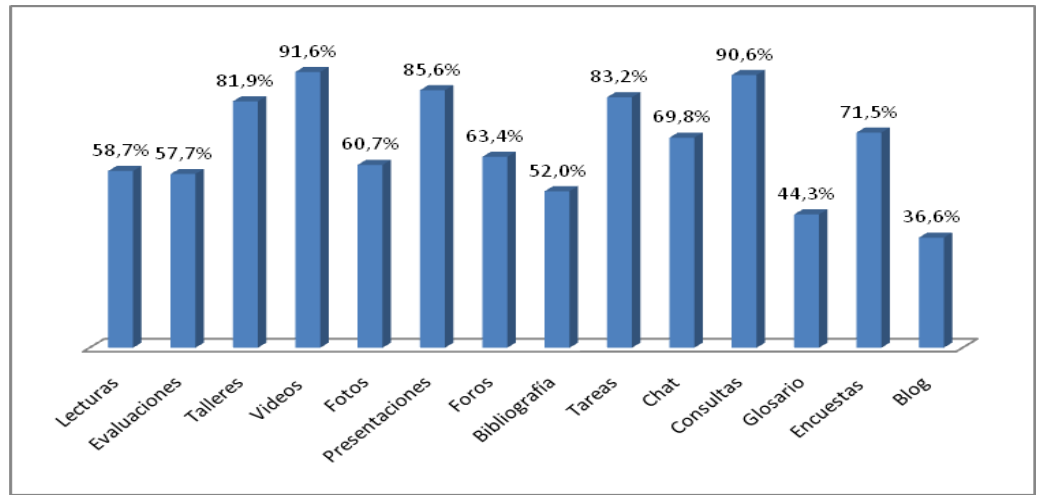

Grafica 1. Actividades académicas desarrolladas en forma virtual.

Tabla 4. Comparativo de la dificultad para el uso de los recursos informáticos por parte de los estudiantes

\begin{tabular}{llcc}
\hline \multicolumn{1}{c}{ Dificultades } & $\begin{array}{c}\text { Frecuencia }-\begin{array}{c}\text { Frecuencia } \\
\text { Encuesta inicial }\end{array} \\
\text { Encuesta final }\end{array}$ \\
\hline a) Complejidad & 20 & 6 \\
b) Disponibilidad de equipos de cómputo & 20 & 20 \\
c) Falta de capacitación & 49 & 30 \\
d) Falta de tiempo & 9 & 6 \\
e) Claridad en la información & 17 & 2 \\
f) Mayor apoyo por parte del docente & 18 & 13 \\
g) Problemas de ingreso a la Plataforma Moodle & 5 & 12 \\
h) Pérdida de comunicación personal con el docente & 13 & 16 \\
i) Velocidad de navegación & 19 & 10 \\
j) Otras & 16 & 12 \\
\hline
\end{tabular}

A manera de síntesis se mencionan los siguientes resultados:

- Se sensibilizó a un grupo de 144 docentes de la Universidad del Quindío en el uso de las TIC aplicadas a la educación superior.

- Se convocó por intermedio de la Vicerrectoría Académica a una capacitación en el uso herramientas informáticas; atendieron el llamado 40 docentes de los cuales 18 profundizaron en el uso de la Plataforma Moodle.

- Se puso a disposición de la comunidad universitaria una instancia de la Plataforma Moodle (22 cursos, 600 estudiantes), así como su entorno de aplicación, que trabaja bajo la filosofía del Open Source. Esta Plataforma permite a los docentes dar continuidad a la capacitación y hacer sus prácticas individuales, antes de poner sus cursos en la Plataforma oficial de la Universidad.

- Se observa el avance en el uso de medios tecnológicos para apoyar la labor docente mediante estrategias bLearning o eLearning en los últimos años como parte de la política institucional, también se observa rechazo cultural por parte de algunos profesores frente al cambio de paradigma, pese a que los estudiantes requieren e impulsan el uso de los medios tecnológicos dada su condición de nativos digitales. Se requiere invertir aún más en capacitación en el uso de recursos informáticos acordes a los requerimientos de la sociedad del conocimiento.

\section{CONCLUSIONES}

Los procesos académicos apoyados por el uso de las TIC, favorecen los procesos enseñanza-aprendizaje, toda vez que permiten potencializar las actividades académicas curriculares y extracurriculares. Sin embargo, para consolidar y/o fortalecer este tipo de metodología mixta, se requiere capacitar a profesores y estudiantes.

La documentación en los temas objeto de estudio, el dialogo permanente con profesores, estudiantes y administrativos, el uso de la Plataforma Moodle y el análisis 
de los instrumentos aplicados, permite llegar a las siguientes conclusiones:

- Es posible utilizar procesos Blended Learning para la formación presencial en la Universidad del Quindío, la mayoría de los profesores reconocen su utilidad en procesos de formación.

- Las herramientas o recursos virtuales no son de uso exclusivo de la formación a distancia y/o virtual, sino que se convierten en un complemento ideal para la formación presencial dada la diversidad de actividades que se pueden realizar en la Plataforma y el interés mostrado por los estudiantes.

- La variedad de cursos que se imparten en los programas académicos, permite diferentes grados de virtualidad, razón fundamental para el uso del bLearning.

- Es posible apoyar los procesos de formación utilizando software libre, dado que reduce considerablemente los costos, además de contar con una comunidad de desarrollo para soportar las aplicaciones dispuesta a asesorar a los usuarios.

- Los estudiantes y profesores de la Institución reclaman la necesidad de formación en el uso de las TIC y en particular, en el manejo de la Plataforma Moodle. Estos últimos están dispuestos a recibir capacitación, virtualizar una parte de sus cursos y en general están interesados en el uso de las TIC como elemento dinamizador del proceso de enseñanza-aprendizaje y de las actividades personales. Aunque la mayoría de los estudiantes pertenecen a la generación llamada nativos digitales, un sector de ellos requiere instruirse en el uso de las TIC

- Actualmente, la Universidad del Quindío ofrece cursos comunes a todos los programas, tales como: Constitución Política, Ética, Creatividad Empresarial y Medio Ambiente. Si se utiliza el bLearning como estrategia de apoyo, la Universidad estará en condiciones de impartir cursos de asistencia masiva (200 o 300 estudiantes de acuerdo a la capacidad de los auditorios). Se recomienda en este caso hacer clases magistrales con expertos, apoyadas con la tutorización virtual en grupos pequeños (tareas, talleres, asesorías, evaluaciones). Este sistema permitiría de un lado unificar criterios en estos cursos y ser asertivos en la relación con el medio, y de otro mejorar la calidad de las conferencias pues se pueden contratar expertos en la materia. Igual sucede en las facultades, pues existen cursos comunes para todos los estudiantes de la facultad.

Finalmente, se invita a toda la comunidad educativa a explorar y utilizar este tipo de metodologías mixtas, toda vez que la mayoría de los estudiantes y de los profesores que participan directamente en esta intervención la califican como buena y piensan que fortalecen los procesos de enseñanza-aprendizaje. En síntesis este es el objetivo principal o una de las razones de ser de la labor docente. 


\section{BIBLIOGRAFIA}

- Brennan, M. (2004). Blended Learning and Business Change. Chief Learning Officer Magazine. Recuperado el 14 de Febrero de 2010, de www.clomedia.com/content/ anmviewer.a sp?a=349

- Bulchand Gidumal, J. (2002). Planes de sistemas y tecnologías de la información y las comunicaciones en las universidades como medio de aprovechamiento del conocimiento: Aplicación al caso de la ULPGC. Tesis doctoral. . Las Palmas de Gran Canaria; España: Universidad de las Palmas de Gran Canaria.

- Carmona Suárez, E. J., \& Gallego Berrío, L. M. (2008). Dashboard digital del docente. Armenia, Quindío: Elizcom.

- Coaten, N. (2003). Blended e-Learning. Recuperado el 2010 de 6 de Septiembre, de Educaweb No 69: www.educaweb.com/esp/servicios/monografico/formacionvirtual/11810 76.asp

- Díaz, M. I. (2008). La educación virtual en la Universidad del Quindío. Dimensión pedagógica. Armenia, Quindío: Universidad del Quindío.

- Druker F, P. (1999). Knowledge Worker productivity: The biggest challenge. California Management Review Vol.1 No 2 , 79-94.

- Fidalgo Blanco, A., \& Ponce, J. (2003). Gestión del conocimiento en las Organizaciones. Madrid España: Universidad Politécnica de Madrid.

- Londoño Orozco, J., \& Díaz Jurado, J. (2008). Incorporación de las TIC en la Universidad del Quindío. Políticas y estrategias . Armenia, Quindío: Universidad del Quindío.

- Marqués Graells, P. (2008). Las TIC en la Educación Social. Bucaramanga, Santander: Universidad Autónoma de Bucaramanga. Colombia.

- Marsh, G. E., McFadden, A. C., \& Price, B. J. (2003). Blended Instruction: adapting Conventional Instruction for Large Classes. Online Journal of Distance Learning Administration, Volume VI, Number IV, Winter 2003.State University of West Georgia, Distance Education Center .

- Murphy, P. (2003). The hybrid strategy: Blending face-to-face with virtual instruction to improve large section courses. Recuperado el 4 de Abril de 2010, de University of California Regents. Teaching, Learning, and Technology Center: www.uctltc.org/news

- Piscitelli, A. (2006). Nativos e inmigrantes digitales: ¿Brecha Generacional, Brecha congnitiva,o las dos juntas y má aun?. RMIE. 11 (18), 179-185.

- Rheingold, H. (2004). Multitudes inteligentes. Barcelona: Gedisa. Cibercultura. Barcelona: Gedisa Cibercultura.

- Rubio Royo, E. (2004). A personal and corporative process-oriented knowledge manager: Suricata model EUNIS. Las Palmas de Gran Canaria, España: CICEI.

- UNESCO. (2005). Hacia las sociedades del conocimiento. Informe mundial de la UNESCO 2005. Organización de las Naciones Unidas para la Educación, la Ciencia y la Cultura. Paris, Francia: UNESCO. 